\title{
Predicting factors of central lymph node metastases in patients with unilateral multifocal papillary thyroid microcarcinoma
}

\author{
Xin Wu, Binglu Li, Chaoji Zheng, Xiaodong He \\ Department of General Surgery, Peking Union Medical College Hospital, Chinese Academy of Medical Sciences and Peking Union Medical \\ College, Beijing 100730, China \\ Contributions: (I) Conception and design: X Wu, B Li; (II) Administrative support: B Li, X He; (III) Provision of study materials or patients: All \\ authors; (IV) Collection and assembly of data: X Wu, B Li, C Zheng; (V) Data analysis and interpretation: Xin Wu, B Li, X He; (VI) Manuscript \\ writing: All authors; (VII) Final approval of manuscript: All authors. \\ Correspondence to: Prof. Binglu Li, MD. Department of General Surgery, Peking Union Medical College Hospital, No.1 Shuaifuyuan, Dongcheng \\ District, Beijing 100730, China. Email: pumchlibinglu@163.com.
}

Background: Unilateral multifocal papillary thyroid microcarcinoma (UM-PTMC) is not rare, and
the suitable extent of operation is controversial. Total thyroidectomy is associated with a high risk of
hypoparathyroidism and recurrent laryngeal nerve injury. Hemithyroidectomy should be considered in
patients without lymph node metastases. This study aimed to identify factors predicting central lymph node
metastases (CLNM) in patients with UM-PTMC.
Methods: A total of 1,453 patients with thyroid cancer underwent surgery at our hospital from January
2018 to June 2018, and 115 patients with UM-PTMC were selected and assessed retrospectively. The
relationship between CLNM and clinicopathologic features of UM-PTMC were analyzed by both univariate
and multivariate analyses. Results: Of the 115 patients with UM-PTMC, CLNM were confirmed by pathology in 57 patients. Univariate and multivariate analyses identified the sum diameter of all tumors $(\geq 1.0 \mathrm{~cm})$ [odds ratio $(\mathrm{OR})$ $=2.295 ; 95 \%$ confidence interval $(\mathrm{CI}): 1.036-5.086 ; \mathrm{P}=0.041]$ and positive CLNM via ultrasonography (OR $=5.882 ; 95 \%$ CI: $1.562-22.153 ; \mathrm{P}=0.009)$ as independent predictors of CLNM.

Conclusions: CLNM are common in patients with UM-PTMC. The sum diameter of all tumors $(\geq 1.0$ $\mathrm{cm}$ ) and positive CLNM via ultrasonography are independent risk factors for CLNM. Our data should be considered in the decision process relating to performing total thyroidectomy or hemithyroidectomy in patients with UM-PTMC.

Keywords: Central lymph node; metastases; multifocal; papillary thyroid microcarcinoma (PTMC); unilateral

Submitted Dec 04, 2019. Accepted for publication Mar 12, 2020.

doi: 10.21037 /gs.2020.03.27

View this article at: http://dx.doi.org/10.21037/gs.2020.03.27

\section{Introduction}

Papillary thyroid carcinoma (PTC) is the most common histological subtype of thyroid cancer. Due to the increased use of diagnostic imaging and fine needle aspiration cytology, the proportion of newly diagnosed PTC has increased significantly in recent years. In China, the incidence of PTC increased more than $30 \%$ annually from 2000 to 2010 (1). This increase is mainly due to an increase in papillary thyroid microcarcinoma (PTMC), which is a type of PTC with a maximum tumor diameter of $10 \mathrm{~mm}(2-4)$.

The suitable extent of operation in patients with PTMC is still controversial, especially for unilateral multifocal papillary thyroid microcarcinoma (UM-PTMC). Some surgeons recommend total thyroidectomy (TT) because it is associated with a low risk of locoregional recurrence. But patients who accept TT would have an increased risk of hypoparathyroidism and recurrent laryngeal nerve 
injury. Another disadvantage of TT is that high-dose lifelong thyroid hormone replacement would be required. Hemithyroidectomy (HT) is an alternative in patients with UM-PTMC, especially for those without lymph node metastases (LNM). The most common site of LNM, the central compartment, has a metastasis frequency of $16.9-35.0 \%$ in PTMC $(1,5)$. Therefore, it is important to identify the predictors of central lymph node metastases (CLNM) in patients with UM-PTMC. This is important for determining the most appropriate surgical approach, for both patients and surgeons. HT is appropriate for UMPTMC without CLNM. The aim of the present study was to identify the predictors of CLNM in patients with UMPTMC.

\section{Methods}

\section{Study design and participants}

A total of 1,453 patients with thyroid cancer underwent surgery at our hospital from January 2018 to June 2018. All the medical records were retrospectively reviewed by two independent doctors. Patients were selected on the basis of the following inclusion criteria: (I) unilateral nodules revealed by preoperative ultrasonography, (II) UM-PTMC diagnosed by postoperative pathology, (III) TT or HT plus central lymph node dissection was performed, and (IV) the medical records of patients were complete. Patients who had previous radiation exposure, thyroid operation history, and coexisting head and neck tumors were excluded. A retrospective database was constructed. Demographic characteristics, thyroid function, ultrasonography outcomes, operation details, and pathological results were reviewed and analyzed. A tumor was defined as multifocal if at least 2 foci were found. The largest lesion was designated as the main focus. The study was approved by the Institutional Review Board of Peking Union Medical College Hospital (S-K1057). The requirement of informed consent for publication of data was waived owing to the retrospective nature of the study.

\section{Treatment}

All patients underwent routine thyroid function test and ultrasonography before operation. Ultrasonography was performed by specially trained sonographers with more than 5 years of experience in thyroid imaging. Prior to surgery, both the benefits and risks of TT and HT were explained by surgeons, and the surgical approach was decided according to the patients' willingness. All patients underwent operation under general anesthesia in the supine position with hypsokinesis of the head. Regardless of whether TT or HT was performed, all patients underwent central compartment lymph node dissection. Lateral neck lymph node dissection was performed only in case where metastases were revealed by preoperative ultrasonography.

Postoperative pathological diagnosis was confirmed by two different pathologists with at least 5 years of experience in thyroid pathology. All patients were postoperatively administered oral euthyrox for suppressive therapy. Thyroid stimulating hormone level was maintained at approximately $0.1 \mu \mathrm{IU} / \mathrm{mL}$. All patients were followed up for at least 1 year postoperatively and underwent ultrasonography every 6 months to exclude tumor residual and recurrence. For patients who underwent HT, the contralateral lobe was examined extremely carefully to exclude any nodules.

\section{Statistical analysis}

The Statistical Package for Social Sciences software (SPSS, version 25.0, IBM Corp., Armonk, NY, USA) was used to perform statistical analysis. Linear variables were presented as mean \pm standard deviation. Categorical variables were described by absolute number or frequency. Differences between study groups were analyzed by the $\chi^{2}$ test or Student's $t$-test as appropriate. Logistic multivariate regression analyses were performed to identify independent predicting factors for CLNM in patients with UM-PTMC. A $P$ value $<0.05$ was considered statistically significant.

\section{Results}

A total of 115 patients with UM-PTMC who underwent surgery from January 2018 to June 2018 were selected (Figure 1). The mean age was $42.2 \pm 10.8$ years (range, 19 72 years). Thirty-four (29.6\%) of the patients were males. One hundred and one $(87.8 \%)$ patients were asymptomatic and incidentally diagnosed with thyroid node. Only $14(12.2 \%)$ patients had symptoms such as hoarseness, dysphagia, and cough. On follow-up, no residual tumor or tumor recurrence was found on ultrasonography performed every 6 months after the operation. For patients who underwent $\mathrm{HT}$, preoperative ultrasonography revealed no contralateral nodule, and the result was confirmed by other ultrasonography every 6 months after surgery.

Based on postoperative pathology, patients were divided 


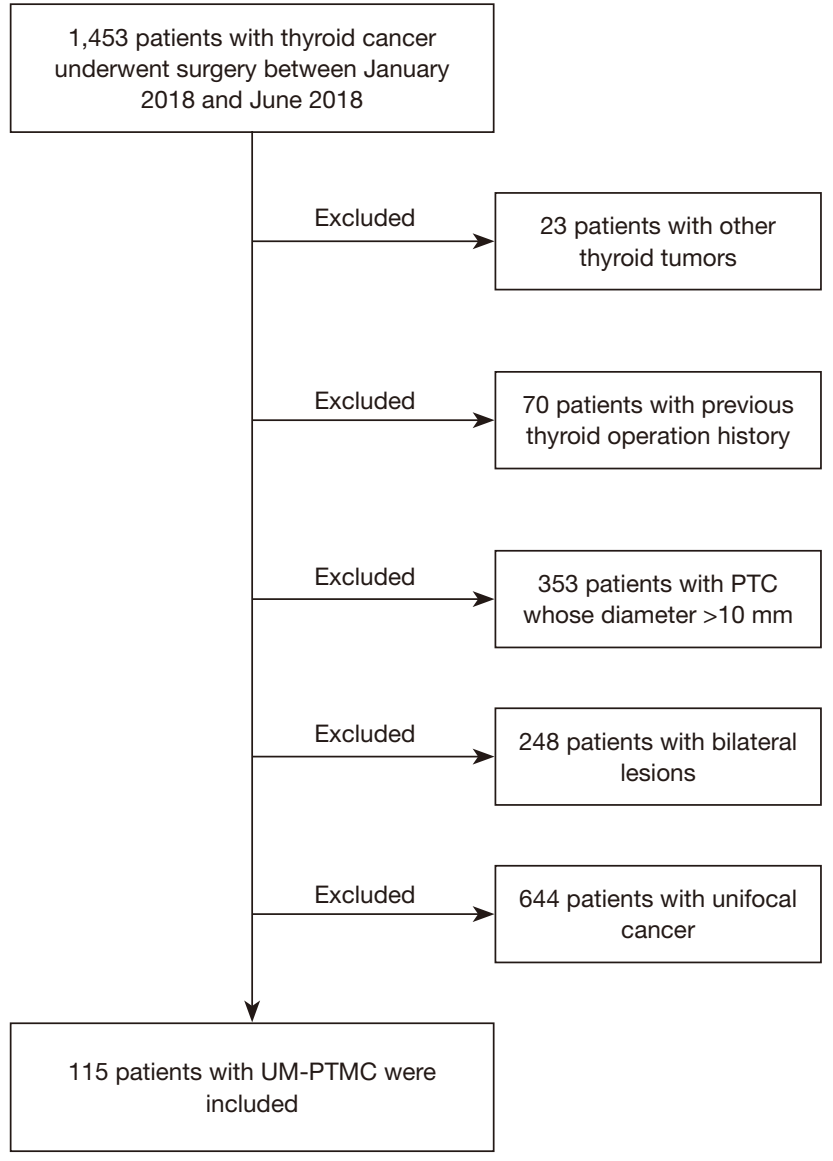

Figure 1 Flow diagram of inclusion criteria of study participants. PTC, papillary thyroid carcinoma; UM-PTMC, unilateral multifocal papillary thyroid microcarcinoma. into two groups: CLNM positive $(\mathrm{n}=57,49.6 \%)$ and CLNM negative $(\mathrm{n}=58,50.4 \%)$. The demographic data and outcomes of thyroid function were analyzed and compared between the two groups (Table 1), and no significant difference was found. Tumors were located in the left lobe in 58 patients and in the right lobe in 57 patients. Mean tumor diameter of the main focus was $0.6 \pm 0.2 \mathrm{~cm}$ (range, $0.1-1.0 \mathrm{~cm}$ ), and mean sum diameter of all foci was $0.9 \pm$ $0.4 \mathrm{~cm}$ (range, $0.2-2.1 \mathrm{~cm}$ ). TT and HT were performed in $69(60.0 \%)$ and $46(40.0 \%)$ patients, respectively. The outcomes of ultrasonography, scope of surgery, and pathological results were compared between the two groups (Table 2). In comparing the CLNM positive and CLNM negative groups, statistically significant differences were found for sum diameter of all foci, extrathyroidal invasion, lateral neck LNM, larger foci number $(\geq 3)$, and positive CLNM via ultrasonography.

Multivariate logistic regression analyses were performed to examine associations between select variables and CLNM. Two variables proved to be independently associated with CLNM. They were larger sum diameter of all foci $(\geq 1.0 \mathrm{~cm})$ and positive CLNM via ultrasonography, with odds ratios of 2.295 and 5.882, respectively (Table 3).

\section{Discussion}

The present study showed that $87.8 \%$ of patients with UMPTMC were asymptomatic, and the rate of CLNM in UMPTMC was $49.6 \%$. A larger sum diameter of all foci $(\geq$

Table 1 Demographic data and thyroid functions of patients with UM-PTMC

\begin{tabular}{|c|c|c|c|c|}
\hline Variables & Total $(n=115)$ & CLNM $(+)(n=57)$ & CLNM $(-)(n=58)$ & $P$ value \\
\hline Age (years) & $42.2 \pm 10.8$ & $41.1 \pm 11.3$ & $43.3 \pm 10.2$ & 0.273 \\
\hline BMI $\left(\mathrm{kg} / \mathrm{m}^{2}\right)$ & $24.7 \pm 3.3$ & $25.0 \pm 3.5$ & $24.4 \pm 3.1$ & 0.315 \\
\hline Thyroid cancer family history (n) & 3 & 1 & 2 & 1.000 \\
\hline FT3 $(\mathrm{pg} / \mathrm{mL})$ & $3.1 \pm 0.5$ & $3.1 \pm 0.5$ & $3.1 \pm 0.5$ & 0.280 \\
\hline FT4 (ng/dL) & $1.3 \pm 0.5$ & $1.2 \pm 0.1$ & $1.3 \pm 0.7$ & 0.565 \\
\hline TSH $(\mu \mathrm{IU} / \mathrm{mL})$ & $2.1 \pm 1.5$ & $2.0 \pm 1.2$ & $2.2 \pm 1.7$ & 0.437 \\
\hline Hashimoto's thyroiditis (n) & 30 & 11 & 19 & 0.100 \\
\hline
\end{tabular}

UM-PTMC, unilateral multifocal papillary thyroid microcarcinoma; CLNM, central lymph node metastases; BMI, body mass index; FT3, free triiodothyronine; FT4, free thyroxine; TSH, thyroid stimulating hormone. 
Table 2 Detailed data regarding operation, ultrasonography, and pathology for patients with UM-PTMC

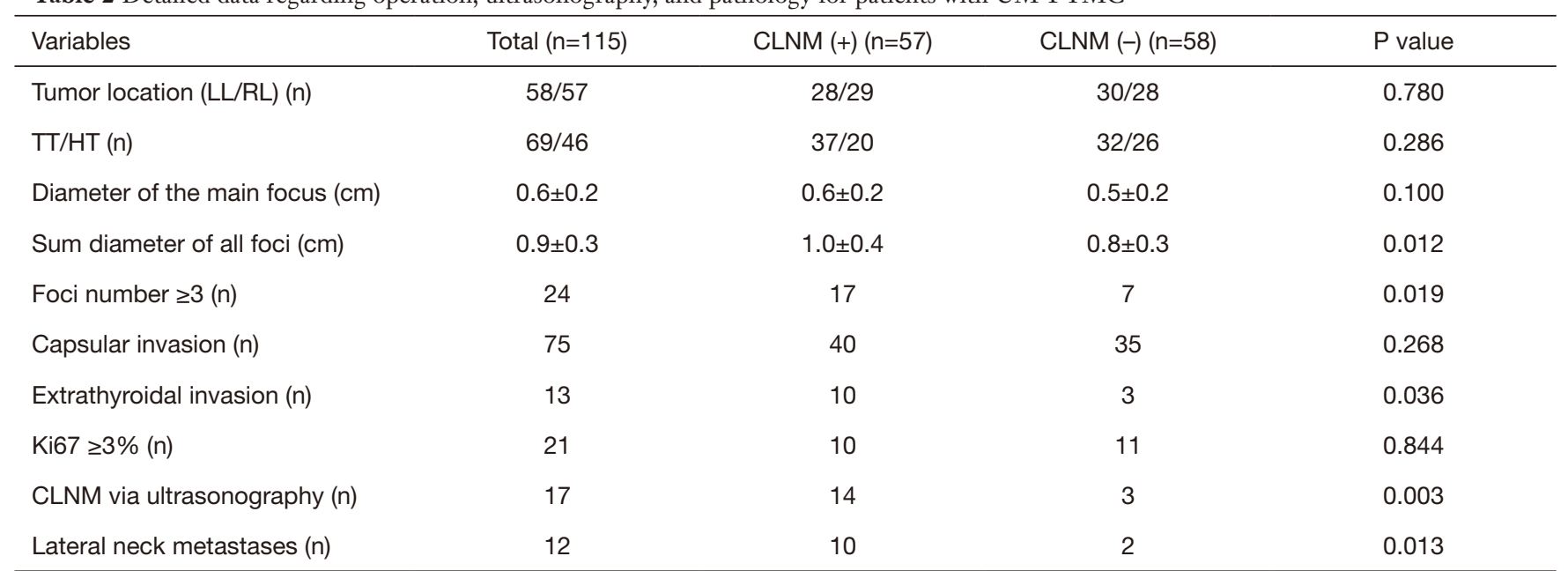

UM-PTMC, unilateral multifocal papillary thyroid microcarcinoma; CLNM, central lymph node metastases; LL, left lobe; RL, right lobe; TT, total thyroidectomy; HT, hemithyroidectomy.

Table 3 Multivariate analyses for predicting factors of CLNM in patients with UM-PTMC

\begin{tabular}{lccc}
\hline Variables & P value & OR & $95 \% \mathrm{Cl}$ \\
\hline Sum diameter of all tumors $(\geq 1.0 \mathrm{~cm})$ & 0.041 & 2.295 & $1.036-5.086$ \\
Positive CLNM via ultrasonography & 0.009 & 5.882 & $1.562-22.153$ \\
Tumor number $(\geq 3)$ & 0.114 & - & - \\
Extrathyroidal invasion & 0.132 & - & - \\
Lateral neck lymph node metastases & 0.322 & -
\end{tabular}

CLNM, central lymph node metastases; UM-PTMC, unilateral multifocal papillary thyroid microcarcinoma; OR, odds ratio; 95\% CI, 95\% confidence interval.

$1.0 \mathrm{~cm}$ ) and positive CLNM via ultrasonography were found to be independently associated with CLNM. As such, we recommend that patients with UM-PTMC who exhibit these two risk factors undergo TT during their initial surgery.

Advancement in ultrasound technology and increased uptake of health examination have resulted in an increase in the diagnosis of PTMC. It was reported that more than $60 \%$ of all thyroid cancers are PTMC (6). Micro tumor means early tumor staging and good prognosis. Conversely, multifocal tumor is associated with more frequent LNM and an increased risk of recurrence $(1,7,8)$. However, an investigation of 2,309 patients by Kim et al. revealed that multifocality was not an independent risk factor for tumor recurrence and persistence in patients with PTMC (9). Therefore, the suitable treatment for UM-PTMC remains controversial, and TT may not be the only option for patients with UM-PTMC. To avoid recurrent laryngeal nerve injury and hypoparathyroidism, HT should be considered in UM-PTMC without LNM. Thus, it is important to determine whether LNM are present before surgical intervention. The preferred method for thyroid examination and detecting the absence of LNM is preoperative ultrasonography, which is affordable and noninvasive. Metastatic lymph nodes often manifest as round shape, cystic change, microcalcification, hypoechogenicity, and hypervascularity (10). Ultrasonography might not detect micro or deep lymph nodes. The sensitivity of preoperative ultrasonography is not high enough and is reported as $13-30 \%(6,11,12)$. A new assessment method for predicting CLNM in patients with UM-PTMC needs to be proposed to assist in determining surgical pattern.

Previous studies have identified risk factors for CLNM in patients with PTMC (13-16). However, very few of 
these studies focused on the predictors of UM-PTMC. The present study clarifies the association between several variables and CLNM in patients with UM-PTMC. Of those variables, larger sum diameter of all foci $(\geq 1.0 \mathrm{~cm})$ and positive CLNM via ultrasonography are proved to be independent risk factors. Larger tumor size contributes to the tumors being more aggressive and is an important factor in tumor staging system. LNM are more frequently found in PTMC with larger tumor size (17-20). Multifocality may arise from multiple independent origins or from intraglandular spread of a single main focus (21-23). Lu et al. used next-generation sequencing technologies and found that $75 \%$ of multicentric PTC cases arose as independent tumors (23). Though it is not included in the tumor staging system, multifocality has been reported as a predictor of CLNM in patients with PTMC $(1,7,18,24)$. UM-PTMC with larger sum diameter of all foci $(\geq 1.0 \mathrm{~cm})$ contains both variables. This could explain why a larger sum diameter of all foci is an independent predictor. Zhao et al. studied 212 patients with PTMC and recommended routine central lymph node dissection in patients with multifocal PTMC with total tumor diameter $>1 \mathrm{~cm} \mathrm{(25).} \mathrm{Liu} \mathrm{et} \mathrm{al.} \mathrm{reported}$ on 1,102 patients with PTC and suggested that total tumor diameter is a relatively more accurate criterion for tumor size of PTC (2). Feng et al. reported 442 patients with PTC and found that multifocal PTMC with a total tumor diameter $>1 \mathrm{~cm}$ was more aggressive than unifocal PTMC or multifocal PTMC with total tumor diameter $\leq 1 \mathrm{~cm}$ (26). Our result was consistent with the results of the abovementioned studies. Though the sensitivity of preoperative ultrasonography in detecting CLNM is low $(24.6 \%, 14 / 57)$, its specificity is high $(82.4 \%, 14 / 17)$ (Table 2). Similar to our study, a specificity of $88.4-95.2 \%$ has been reported by previous studies $(12,27)$. This may be the reason why positive CLNM via ultrasonography could be a potential risk factor.

Between the CLNM positive and negative groups, several variables show significant differences during univariate analysis but are not significant in multivariate analysis. These include tumor number $(\geq 3)$, extrathyroidal invasion, and lateral neck LNM. Some previous studies also reported that young age ( $<45$ years), male gender, and lymphovascular invasion could be the predictors of LNM $(1,24,28)$. In the present study, no significant association was found between the abovementioned variables and CLNM. A possible explanation could be that UM-PTMC has characteristics that differ from those of other thyroid cancers and that the sample size was small.
At present, prophylactic lymph node dissection is recommended in patients with multifocal thyroid cancer $(10,29)$. Xue et al. suggested TT in UT-PTMC because it had a lower recurrence rate than that of HT (30). However, it was reported that multifocality was not an independent predictor of CLNM (20) and had no association with mortality (31). Choi et al. retrospectively reviewed 2,390 patients with PTC and found that the prognostic value of multifocality was more significant in PTC of $>1 \mathrm{~cm}$ tumor size than in PTMC (32). Thus, the best approach to manage patients with UT-PTMC remains controversial. The debate centers on whether it is necessary to remove the contralateral lobe which would lead to more complications, such as nerve damage and hypoparathyroidism, while conservative treatment would lead to an increased recurrence rate. Based on our findings, larger sum diameter of all foci $(\geq 1.0 \mathrm{~cm})$ and positive CLNM via ultrasonography are independent risk factors of CLNM in patients with UM-PTMC, and we recommend TT in UMPTMC patients with these two variables.

There are some limitations of this study. First, because of its retrospective nature, the patient volume, registration information, and inspection items could not be designed beforehand. Second, some patients underwent HT and the unilateral feature was confirmed by ultrasonography. Subclinical contralateral microcarcinoma might exist and result in wrong grouping. Third, the duration of follow-up was short. To overcome these limitations and provide more reliable data, prospective, randomized, controlled, and multicenter clinical trials are necessary.

In conclusion, our results suggest that the sum diameter of all tumors $(\geq 1.0 \mathrm{~cm})$ and positive CLNM via ultrasonography are independent factors for CLNM in patients with UM-PTMC. Therefore, these factors should be considered by surgeons when deciding whether to perform TT in patients with UM-PTMC.

\section{Acknowledgments}

We thank all the patients who participated in this study. Funding: This study is supported by the Fundamental Research Funds for the Central Universities of China (3332019022).

\section{Footnote}

Conflicts of Interest: All authors have completed the ICMJE uniform disclosure form (available at http://dx.doi. 
org/10.21037/gs.2020.03.27). The authors have no conflicts of interest to declare.

Ethical Statement: The authors are accountable for all aspects of the work in ensuring that questions related to the accuracy or integrity of any part of the work are appropriately investigated and resolved. The study was approved by the Institutional Review Board of Peking Union Medical College Hospital (S-K1057). The requirement of informed consent for publication of data was waived owing to the retrospective nature of the study.

Open Access Statement: This is an Open Access article distributed in accordance with the Creative Commons Attribution-NonCommercial-NoDerivs 4.0 International License (CC BY-NC-ND 4.0), which permits the noncommercial replication and distribution of the article with the strict proviso that no changes or edits are made and the original work is properly cited (including links to both the formal publication through the relevant DOI and the license). See: https://creativecommons.org/licenses/by-nc$\mathrm{nd} / 4.0 \%$.

\section{References}

1. Xu Y, Xu L, Wang J. Clinical predictors of lymph node metastasis and survival rate in papillary thyroid microcarcinoma: analysis of 3607 patients at a single institution. J Surg Res 2018;221:128-34.

2. Liu C, Wang S, Zeng W, et al. Total tumour diameter is superior to unifocal diameter as a predictor of papillary thyroid microcarcinoma prognosis. Sci Rep 2017;7:1846.

3. Kang JG, Kim YA, Choi JE, et al. Usefulness of 1 -year of thyroid stimulating hormone suppression on additional levothyroxine in patients who underwent hemithyroidectomy with papillary thyroid microcarcinoma. Gland Surg 2019;8:636-43.

4. Jeon MJ, Kim WG, Chung KW, et al. Active surveillance of papillary thyroid microcarcinoma: where do we stand? Eur Thyroid J 2019;8:298-306.

5. Liu C, Liu Y, Zhang L, et al. Risk factors for highvolume lymph node metastases in $\mathrm{cN} 0$ papillary thyroid microcarcinoma. Gland Surg 2019;8:550-6.

6. Wu X, Li B, Zheng C, et al. Risk factors for central lymph node metastases in patients with papillary thyroid microcarcinoma. Endocr Pract 2018;24:1057-62.

7. Gui CY, Qiu SL, Peng ZH, et al. Clinical and pathologic predictors of central lymph node metastasis in papillary thyroid microcarcinoma: a retrospective cohort study. J Endocrinol Invest 2018;41:403-9.

8. Zhang Q, Wang Z, Meng X, et al. Predictors for central lymph node metastases in $\mathrm{CN} 0$ papillary thyroid microcarcinoma (mPTC): A retrospect analysis of 1304 cases. Asian J Surg 2019;42:571-6.

9. Kim KJ, Kim SM, Lee YS, et al. Prognostic significance of tumor multifocality in papillary thyroid carcinoma and its relationship with primary tumor size: a retrospective study of 2,309 consecutive patients. Ann Surg Oncol 2015;22:125-31.

10. Haugen BR, Alexander EK, Bible KC, et al. 2015 American Thyroid Association management guidelines for adult patients with thyroid nodules and differentiated thyroid cancer: the American Thyroid Association guidelines task force on thyroid nodules and differentiated thyroid cancer. Thyroid 2016;26:1-133.

11. Yu X, Song X, Sun W, et al. Independent risk factors predicting central lymph node metastasis in papillary thyroid microcarcinoma. Horm Metab Res 2017;49:201-7.

12. Huang XP, Ye TT, Zhang L, et al. Sonographic features of papillary thyroid microcarcinoma predicting highvolume central neck lymph node metastasis. Surg Oncol 2018;27:172-6.

13. Zheng $\mathrm{W}$, Wang $\mathrm{K}, \mathrm{Wu} \mathrm{J}$, et al. Multifocality is associated with central neck lymph node metastases in papillary thyroid microcarcinoma. Cancer Manag Res 2018;10:1527-33.

14. Wang Y, Guan Q, Xiang J. Nomogram for predicting central lymph node metastasis in papillary thyroid microcarcinoma: A retrospective cohort study of 8668 patients. Int J Surg 2018;55:98-102.

15. Akın Ş, Yazgan Aksoy D, Akın S, et al. Prediction of central lymph node metastasis in patients with thyroid papillary microcarcinoma. Turk J Med Sci 2017;47:1723-7.

16. Oh HS, Park S, Kim M, et al. Young age and male sex are predictors of large-volume central neck lymph node metastasis in clinical N0 papillary thyroid microcarcinomas. Thyroid 2017;27:1285-90.

17. Jin WX, Ye DR, Sun YH, et al. Prediction of central lymph node metastasis in papillary thyroid microcarcinoma according to clinicopathologic factors and thyroid nodule sonographic features: a case-control study. Cancer Manag Res 2018;10:3237-43.

18. Luo Y, Zhao Y, Chen K, et al. Clinical analysis of cervical lymph node metastasis risk factors in patients with papillary thyroid microcarcinoma. J Endocrinol Invest 2019;42:227-36. 
19. Cheng F, Chen Y, Zhu L, et al. Risk factors for cervical lymph node metastasis of papillary thyroid microcarcinoma: a single-center retrospective study. Int J Endocrinol 2019;2019:8579828.

20. Zheng X, Peng C, Gao M, et al. Risk factors for cervical lymph node metastasis in papillary thyroid microcarcinoma: a study of 1,587 patients. Cancer Biol Med 2019;16:121-30.

21. Mazeh H, Samet Y, Hochstein D, et al. Multifocality in well-differentiated thyroid carcinomas calls for total thyroidectomy. Am J Surg 2011;201:770-5.

22. Sun W, Lan X, Zhang H, et al. Risk factors for central lymph node metastasis in CN0 papillary thyroid carcinoma: a systematic review and meta-analysis. PLoS One 2015;10:e0139021.

23. Lu Z, Sheng J, Zhang Y, et al. Clonality analysis of multifocal papillary thyroid carcinoma by using genetic profiles. J Pathol 2016;239:72-83.

24. Yuan J, Li J, Chen X, et al. Identification of risk factors of central lymph node metastasis and evaluation of the effect of prophylactic central neck dissection on migration of staging and risk stratification in patients with clinically node-negative papillary thyroid microcarcinoma. Bull Cancer 2017;104:516-23.

25. Zhao Q, Ming J, Liu C, et al. Multifocality and total tumor diameter predict central neck lymph node metastases in papillary thyroid microcarcinoma. Ann Surg Oncol

Cite this article as: $\mathrm{Wu} \mathrm{X,} \mathrm{Li} \mathrm{B,} \mathrm{Zheng} \mathrm{C,} \mathrm{He} \mathrm{X.} \mathrm{Predicting}$ factors of central lymph node metastases in patients with unilateral multifocal papillary thyroid microcarcinoma. Gland Surg 2020;9(3):695-701. doi: 10.21037/gs.2020.03.27
2013;20:746-52.

26. Feng JW, Pan H, Wang L, et al. Total tumor diameter: the neglected value in papillary thyroid microcarcinoma. J Endocrinol Invest 2020;43:601-13.

27. Lee YJ, Kim DW, Park HK, et al. Pre-operative ultrasound diagnosis of nodal metastasis in papillary thyroid carcinoma patients according to nodal compartment. Ultrasound Med Biol 2015;41:1294-300.

28. Liu LS, Liang J, Li JH, et al. The incidence and risk factors for central lymph node metastasis in $\mathrm{cN} 0$ papillary thyroid microcarcinoma: a meta-analysis. Eur Arch Otorhinolaryngol 2017;274:1327-38.

29. Sancho JJ, Lennard TW, Paunovic I, et al. Prophylactic central neck disection in papillary thyroid cancer: a consensus report of the European Society of Endocrine Surgeons (ESES). Langenbecks Arch Surg 2014;399:155-63.

30. Xue S, Wang P, Liu J, et al. Total thyroidectomy may be more reasonable as initial surgery in unilateral multifocal papillary thyroid microcarcinoma: a single-center experience. World J Surg Oncol 2017;15:62.

31. Wang F, Yu X, Shen X, et al. The prognostic value of tumor multifocality in clinical outcomes of papillary thyroid cancer. J Clin Endocrinol Metab 2017;102:3241-50.

32. Choi WR, Roh JL, Gong G, et al. Multifocality of papillary thyroid carcinoma as a risk factor for disease recurrence. Oral Oncol 2019;94:106-10. 